\title{
DETERMINATION OF THERMAL LOAD IN FILM COOLED BIPROPELLANT THRUST CHAMBERS BY AN INVERSE METHOD
}

J. N. Hinckel, R. I. Savonov, and H. Patire, Jr.

INPE

Sao José dos Campos, SP, Brasil

\begin{abstract}
A method to obtain the heat load on the internal wall of a rocket thrust chamber using an inverse problem approach is described. According to the "classical" approach, the heat load on the internal wall of the chamber is assumed as the product of a heat transfer coefficient and the temperature difference of adiabatic wall temperature and local wall surface temperature. The time-dependent temperature distribution of the external wall of the thruster chamber is used to obtain empirical curve fittings to the temperature profile of the near wall flow field (adiabatic wall temperature) and the heat transfer coefficient profile. The applicability of the method is verified by applying it to three different problems; a model problem, an analytical solution, and a set of experimental data.
\end{abstract}

\section{INTRODUCTION}

The evaluation of the heat load applied to the internal wall of the thrust chamber of rocket engines presents a formidable challenge to a designer of these machines, even for long time practitioners of the art. The analytical tools available for the task are limited by the difficulty of modeling the underlying physical phenomena: the atomization, the evaporation, the mixing and the combustion of the propellants, and the flow of the resulting gas mixture.

The discreet nature of the injection process, the minimal chamber volume and the high energy release in the entire volume of the chamber renders meaningless the assumptions of the "classical" analytical solution. The attempts of the numerical solution of the problem are also adversely affected by the same problems.

This is an Open Access article distributed under the terms of the Creative Commons Attribution License 2.0, which permits unrestricted use, distribution, and reproduction in any medium, provided the original work is properly cited. 


\section{DESCRIPTION OF THE METHOD}

The method described here is intended to be used as a tool to obtain the heat load on the inner surface of the thrust chamber of a rocket engine from the mapping of the temperature of the external surface of the chamber. To the degree that the heat load depends only on the flow of the combustion products and that the transient regime of the flow is very short compared to the heating of the chamber wall, data collected during a short test can be used to determine the heat load. In a radiatively cooled chamber, the heat load data can be used to evaluate a steady-state temperature distribution of the chamber for different materials and wall thicknesses. For heat sink cooled chambers, the heat load solution can be used to distribute the heat sinking power along the surface of the wall chamber.

\subsection{Underlying Hypotheses}

The basic assumption of this approach is to circumvent the difficulty of direct measurement of the heat load applied to the inner surface of the thrust chamber. It is reasonably easy to measure the outside wall temperature and it is possible to model accurately the transfer function relating the convective and radiative heat load applied on the inner wall and a radiating outer wall.

The inverse problem cannot be solved exactly, but, combining the modeling of the conduction transfers across the wall and some reasonable assumptions about the temperature of the combustion products and the gas flow near the wall, it is possible to obtain a good estimation of the heat load on the inner wall.

The main hypotheses regarding the temperature of the combustion products and the gas flow near the wall are:

- the characteristic time for the establishment of a steady state flow inside the combustion chamber is much smaller than the time constant for heating of the chamber wall; and

- the heat flux from the combustion products to the chamber wall is described with a good accuracy by the product of the heat transfer coefficient and the temperature difference between the wall surface and the near wall film temperature (represented by the adiabatic wall temperature).

To the extent that the above hypotheses hold, the heat load to the thrust chamber can be determined with a short test in a transient time. The results may be used to extrapolate the wall temperature profile in the steady state. The method may be used to evaluate injector head layouts and chamber geometries (with respect to the heat load), using less expensive materials and surface treatment methods to produce thrust chambers capable of operating at very high temperatures. 
The method might also be used to evaluate the heat load even in regeneratively cooled chambers. An uncooled thrust chamber would be used to determine the inside wall heat load. The wall temperature data would be accquired during the transient heating. The duration of the tests is limited by the wall heat load, the heat capacity of the chamber and the temperature dependent mechanical properties of the test wall material. By applying the inverse method, the heat load (which is only weakly dependent on the wall temperature) is obtained. The detailed knowledge of the heat load will help in designing the distribution of the heat sinking profile of the cooling channels along the length of the thrust chamber.

\subsection{Governing Equations}

The heat load on the internal surface of the chamber wall is given by:

$$
q(x, t)=h(x)\left(T_{a w}(x)-T_{w}(x, t)\right)
$$

Here, the expressions $h(x)$ and $T_{a w}(x)$ depend only on conditions of the flow inside the chamber and, according to the basic assumptions, depend only on the distance along the axis of the chamber. The characteristic time of these profiles is determined by the combustion process of the propellants. Temperature $T_{w}(x, t)$ is the temperature of the inner surface of the thrust chamber and depends on the axial coordinate and the time. The temperature of the wall is governed by the transient heat conduction problem with the initial condition of homogeneous temperature distribution, a convective boundary condition on the inside surface and the radiative condition on the outside wall. On the inside wall there is also a heat flux due to radiative exchange with the hot gases.

The equations describing this problem are:

$$
\nabla[k \nabla T(\mathbf{r}, t)]=\rho C_{p} \frac{\partial T(\mathbf{r}, t)}{\partial t} .
$$

The initial condition is:

$$
\left.T(\mathbf{r}, t)\right|_{t=0}=T_{0} .
$$

The boundary condition on the inside wall (without the radiative exchange) is:

$$
q(\mathbf{r}, t)=h(\mathbf{r}, t)\left[T_{a w}(\mathbf{r})-T_{w}(\mathbf{r}, t)\right] .
$$

The boundary condition on the outside wall is given by

$$
q(\mathbf{r}, t)=\varepsilon \sigma\left\{\left[T_{w}(\mathbf{r}, t)\right]^{4}-T_{\infty}^{4}\right\} .
$$




\subsection{Temperature Profile of the Near Wall Flow Field}

The temperature of the near wall flow film depends on several factors: the amount of a fuel injected in the curtain, the heat absorbed by the evaporation and the heating of the fuel film, the heat lost to the wall and the heat released by the combustion of the fuel in the film with an oxidizer migrating from the core flow. As a result of all these phenomena the temperature of the film is generally increasing from the face of the injector plate towards the exit of the nozzle. The temperature of the film is also limited by two obvious asymptotes; the entrance temperature of the film and the adiabatic wall temperature of the core flow.

A Boltzmann profile curve has the characteristics described above. This curve is described by the following equation:

$$
T(x)=\frac{T_{1}-T_{2}}{1+e^{\left(x-x_{0}\right) / \Delta x}}+T_{2}
$$

where variables $T_{1}$ and $T_{2}$ are the asymptotes; $x_{0}$ is the axial coordinate of the mean value of the asymptotes; and $\Delta x$ is the "width" of the growth rate at the $x_{0}$ coordinate.

By changing the values of these four parameters, a large family of curves may be obtained.

\subsection{Heat Transfer Coefficient Profile}

The heat transfer coefficient profile may be obtained in an approximated form by the Bartz formula.

The Bartz formula contains many parameters related to the geometry of the chamber flow profile of the combustion products inside the thrust and thermophysical and transport properties of combustion products. The strongest dependency is on the mass flux which may be expressed via the ratio of the area of the cross section of the chamber in each position along the length of the chamber to the area of the cross section of the chamber at the throat of the nozzle.

The general behavior of the heat transfer coefficient along the length of the chamber is an increase from the injector until the throat and a decrease thereafter.

A type of curve that presents this general behavior is the Lorentz curve described by the following formula:

$$
L(x)=\frac{1}{\pi} \frac{\Gamma / 2}{\left(x-x_{0}\right)^{2}+(\Gamma / 2)^{2}} .
$$


The Lorentz curve has the following characteristics:

- the area under the curve is unitary:

$$
\int_{-\infty}^{\infty} L(x)=1
$$

- the function has a maximum at $x=x_{0}$ given by:

$$
L\left(x_{0}\right)=\frac{2}{\pi \Gamma}
$$

where $\Gamma$ is the the Full Width at Half Maximum (FWHM).

The formula is redefined so that the horizontal asymptote is $H_{0}$ and the maximum is $H_{0}+H_{1}$. To account for the fact that the heat transfer coefficient is not even with respect to the throat axial coordinate, two sets of parameters are used, one for the combustion chamber and the subsonic part of the nozzle, $L_{l}(x)$, and one for the supersonic part of the nozzle, $L_{r}(x)$ :

$$
\begin{aligned}
& L_{l}(x)=H_{l_{0}}+\frac{H_{l_{1}} W_{l}^{2}}{4\left[\left(x-x_{l_{0}}\right)^{2}+W_{l}^{2} / 4\right]} ; \\
& L_{r}(x)=H_{r_{0}}+\frac{H_{r_{1}} W_{r}^{2}}{4\left[\left(x-x_{r_{0}}\right)^{2}+W_{r}^{2} / 4\right]} .
\end{aligned}
$$

The combination of the above equations to form a single profile may be done in different ways. The transition occurs near the throat and may be done at a single coordinate point or we can think of a smooth transition over a discrete interval.

A sharp transition at the point $x_{l_{0}}$ is chosen. Further, it is required that the profile be continuous and has no inflexion point near the transition. This condition is satisfied if additional equations are set for the parameters of the left portion (subsonic) and right portion (supersonic) of the curve $x_{l_{0}}=x_{r_{0}}=x_{t}$ and $H_{r_{1}}=H_{l_{0}}+H_{l_{1}}-H_{r_{0}}$. This condition reduces by 2 units the number of free parameters of the curve fittings:

$$
h(x)= \begin{cases}L_{l}(x) & \text { if } x \leq x_{t} \\ L_{r}(x) & \text { if } x>x_{t}\end{cases}
$$

Figure 1 shows the Boltzmann type temperature profile for the near wall film and the Lorentz type heat transfer coefficient profile. This family of curves is large but limited, and certainly does not constitute a base space for all possible profiles. 


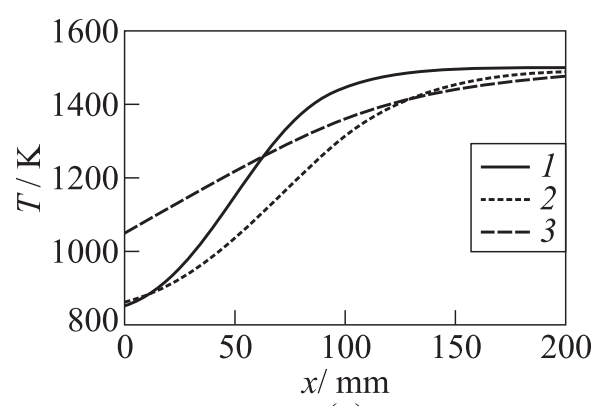

(a)

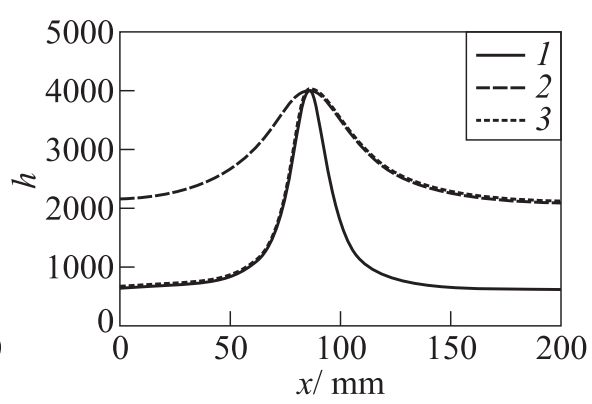

(b)

Figure 1 Boltzmann near-wall temperature profiles at different parameters: 1 $x_{0}=0.05$ and $d x=0.02 ; 2-x_{0}=0.07$ and $d x=0.03 ; 3-x_{0}=0.03$ and $d x=0.05$; $T_{1}=800 \mathrm{~K}$; and $T_{2}=1500 \mathrm{~K}(a)$ and Lorentz heat transfer coefficients profiles $(b)$ $\left(1-H_{l} ; 2-H_{r} ;\right.$ and $\left.3-h\right)$

\section{ALGORITHM}

Equations (1) and (2) define the thermal load on the inner surface of the thrust chamber. Ten free parameters of the heat load are determined by minimizing the difference between the measured temperature on the outside surface of the wall and the temperature profile obtained from the solution of the transient heat conduction in the wall with the applied heat load.

The transient heat conduction problem for the thrust chamber wall is solved numerically using a finite element based application.

The "merit function" to be minimized is given by

$$
\chi^{2}(\mathbf{a})=\sum_{i=1}^{N}\left[y_{i}-y\left(x_{i} ; \mathbf{a}\right)\right]^{2}
$$

where $y_{i}$ is the vector of the measured temperature values along the external surface and $y\left(x_{i} ; \mathbf{a}\right)$ is the vector of temperature values at the corresponding points obtained from the "test" heat load. In the solution presented here, the merit function is evaluated at one single time point during the transient or in the steady state. The merit function could also be defined based on the difference of the temperatures summed over different time intervals.

Vector $\mathbf{a}$ is defined by

$$
\mathbf{a}=\left\{T_{1}, T_{2}, x_{0}, \Delta x, H_{l_{0}}, H_{l_{1}}, x_{t}, W_{l}, H_{r_{0}}, W_{r}\right\} .
$$

The algorithm due to Marquardt, usually referred to as the LevenbergMarquardt method, described in [1], is used in the iterative process to obtain vector $\mathbf{a}$. 


\section{VALIDATION}

The validation of the method was done in various steps.

\subsection{Model Problem}

In the first step, the method was verified for consistency. A set of "reasonable" values of the heat load parameters was assumed (i. e., the vector a was populated). With this "reasonable" heat load, the transient heat conduction problem in the chamber wall was solved and the temperature profile on the external wall surface was obtained in several steps during the transient heating of the wall and in the steady state. The initial condition was homogeneous temperature. External surface boundary condition was radiation to empty medium. These data were fed into the method to recover the heat load parameters.

The whole sequence of jobs is as follows:

- choose a value of vector $\mathbf{a}$;

- solve the problem for transient time and in steady-state regime; and

- compare capability of method to capture initial values of the vector a in the transient time and in the steady state.

Two wall geometries were used. In the first geometry, the wall thickness was uniform. As the thickness of the wall decreases, the axial conduction along the wall can be neglected and the heat resistance is due only to the convection and radiation. In the second geometry, the wall thickness of the thrust chamber varies along the length of the chamber. The internal geometry of both chambers is the same. The variation of the wall thickness is obtained by changing the external geometry of the chamber. The geometries of the chamber are presented in Fig. 2. The thickness of the uniform wall was $1 \mathrm{~mm}$. The thickness of the variable wall varied from 1 to $6 \mathrm{~mm}$. The material thermophysical properties used in the solution were those of Inconel 600 .

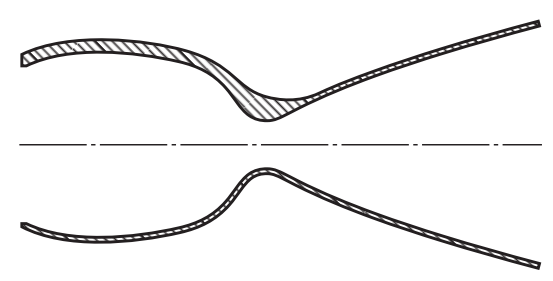

Figure 2 Wall chamber geometries: top half — variable thickness wall chamber; and bottom half - uniform thickness wall chamber 
Table 1 Reference and recovered vectors in steady state (SS) and during transient time in 1- (1s) and 5-second (5s) intervals

\begin{tabular}{|c|c|c|c|c|c|c|c|c|c|c|c|}
\hline Vector & $T_{1}, \mathrm{~K}$ & $T_{2}, \mathrm{~K}$ & $\begin{array}{l}x_{0}, \\
\mathrm{~mm}\end{array}$ & $\begin{array}{l}\Delta x, \\
m m\end{array}$ & $\begin{array}{c}H_{l_{0}} \\
\mathrm{~W} /\left(\mathrm{m}^{2} \mathrm{~K}\right)\end{array}$ & $\begin{array}{c}H_{l_{1}} \\
\mathrm{~W} /\left(\mathrm{m}^{2} \mathrm{~K}\right)\end{array}$ & $\begin{array}{l}x_{t}, \\
\mathrm{~mm}\end{array}$ & $\begin{array}{l}W_{l}, \\
\mathrm{~mm}\end{array}$ & $\begin{array}{c}H_{r_{0}} \\
\mathrm{~W} /\left(\mathrm{m}^{2} \mathrm{~K}\right)\end{array}$ & $\begin{array}{c}H_{r_{1}} \\
\mathrm{~W} /\left(\mathrm{m}^{2} \mathrm{~K}\right)\end{array}$ & $\begin{array}{l}W_{r}, \\
\mathrm{~mm}\end{array}$ \\
\hline Reference & 800 & 1500 & 50 & 20 & 600 & 3400 & 86.0 & 20 & & 3500 & 50 \\
\hline Guess & 550 & 2000 & 35 & 14 & 780 & 4000 & 90.0 & 26 & 280 & 4500 & 65 \\
\hline \multicolumn{12}{|c|}{ Uniform wall thickness } \\
\hline Recovered: SS & 800 & 1500 & 50 & 20 & 598.4 & 3388 & 86.2 & 20 & 498 & 3488 & 50 \\
\hline Recovered: $5 \mathrm{~s}$ & 800.4 & 1499.4 & 50 & 20 & 599 & 3407 & 86.2 & 20 & 501 & 3505 & 50 \\
\hline Recovered: $1 \mathrm{~s}$ & 800 & 1500 & 50 & 20 & 600 & 3397 & 86.2 & 20 & 499 & 3498 & 50 \\
\hline \multicolumn{12}{|c|}{ Variable wall thickness } \\
\hline Recovered: SS & 800 & 1500 & 50 & 20 & 600.4 & 3400.4 & 86.2 & 20 & 500 & 3500 & 50 \\
\hline Recovered: 5s & 800 & 1500 & 50 & 20 & 600 & 3400 & 86.2 & 20 & 500 & 3500 & 50 \\
\hline Recovered: 1s & 690.4 & 1606 & 65 & 26 & 727 & 3627 & 86.2 & 21 & 446 & 3908 & 46 \\
\hline
\end{tabular}




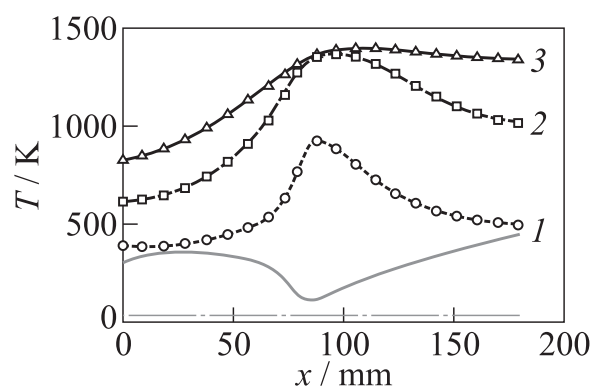

(a)

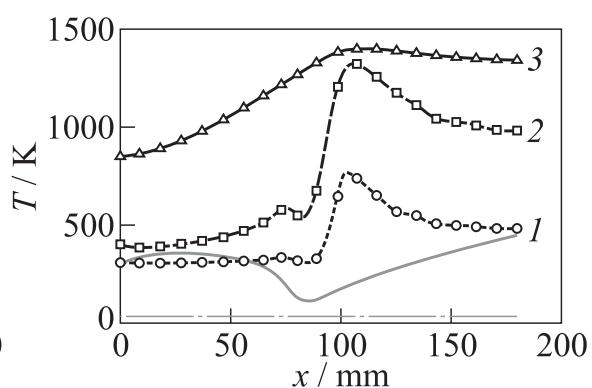

(b)

Figure 3 External surface temperature from the reference and recovered data: two geometries, transient time $(1-1 \mathrm{~s}$ and $2-5 \mathrm{~s})$ and steady state $(3)$ : uniform $(a)$ and variable $(b)$ wall thicknesses

The convergence of the iterative process is sensitive to the "closeness" of the guess vector. If the guess vector is too far off from the solution, the iterative process may not converge at all. When convergence is achieved, the number of iterations is in the range of 20 to 100, depending on the desired accuracy and closeness of the guess vector to the solution. It should be noted that in this model problem, the imposed heat load profile matches the fitting curves. Therefore, the exact value of the solution exists and, in principle, may be obtained.

As expected, the algorithm was able to recover the heat load data with a great accuracy. The results for the two geometries are shown in Table 1. The external wall temperature profile for the reference data and recovered data are in a very good agreement except for the case of the variable wall thickness with reference data from the interval of $1 \mathrm{~s}$ in the transient heating of the wall.

The external surface temperature calculated for the reference heat load and the recovered data heat load is shown in Fig. 3. The surface temperature is plotted for the two geometries during the transient time in the intervals of 1 and $5 \mathrm{~s}$, and in the steady state. Each curve is composed of a continuous line and supperimposed staggered symbols. The continuous line refers to the corresponding heat load condition. The staggered symbols refers to the recovered heat load data at the same time interval and chamber geometry.

It is interesting to note that even for the case of variable wall thickness in the 1-second interval transient condition, there is a very good agreement between the curves for the reference heat load and the recovered data heat load. This suggests that the obtained profiles for the heat transfer coefficient and the temperature difference are not "orthogonal" under these conditions.

In Fig. 4, the near-wall gas temperature profile, the internal and external wall surface temperature profiles, and the heat flux on the internal and external 


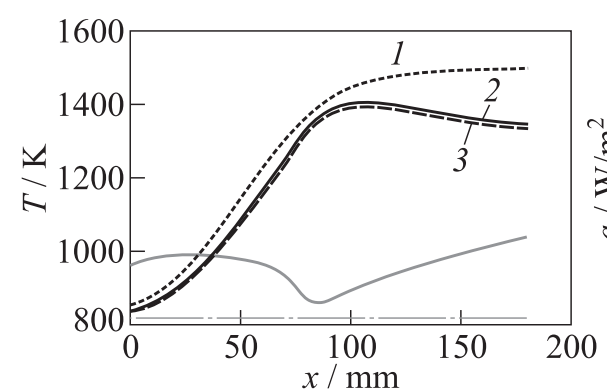

(a)

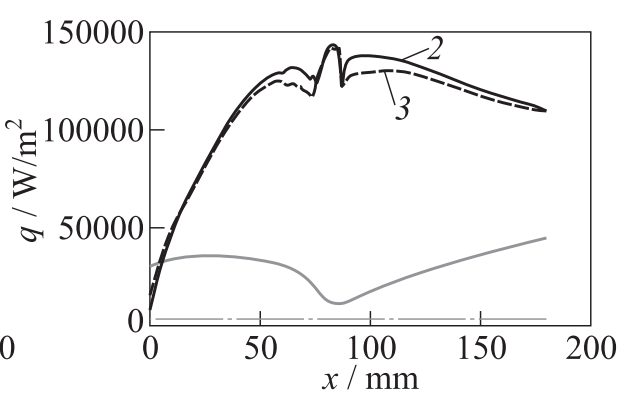

(b)

Figure 4 Gas (1) and internal (2) and external wall (3) temperatures $(a)$ and heat flux $(b)$. Steady-state conditions; uniform wall thickness

wall surfaces are shown. These data refer to the uniform wall thickness chamber in the steady state.

\subsection{Bartz Solution}

In the second step, the temperature profile of the near wall flow field was assumed to be given by the stagnation temperature of the combustion product, corrected along the length of the chamber by the recovery factor. The heat transfer coefficient was calculated by the Bartz formula. A description of the Bartz method is presented in $[2,3]$. The purpose of this step was to show that the chosen near wall temperature profile and heat transfer coefficient profile could represent reasonably well "real world" conditions.

The Bartz solution assumes a fully mixed and a fully reacted core flow inside the thrust chamber. Under these conditions the stagnation temperature is constant along the whole length of the chamber. The core temperature actually "seen" by the wall, the adiabatic wall temperature, is slightly decreasing due to a recovery factor smaller than 1.0. In real engines, the physical processes of evaporation, atomization, mixing and combustion take place along the length of the subsonic part of the chamber. By design, the mixing of the near wall protective layer with the core flow may extend beyond the throat region. If the mixing and the reaction of the near wall layer with the core flow are modeled via a diffusive process, the resulting value of the near wall temperature increases according to a profile that may be represented reasonably well by a Boltzmann type curve.

The purpose of including the Bartz solution in the validation of the model is to show that the method is robust enough to capture the heat load even under these condition. A fully mixed and fully reacted nozzle expanded flow 

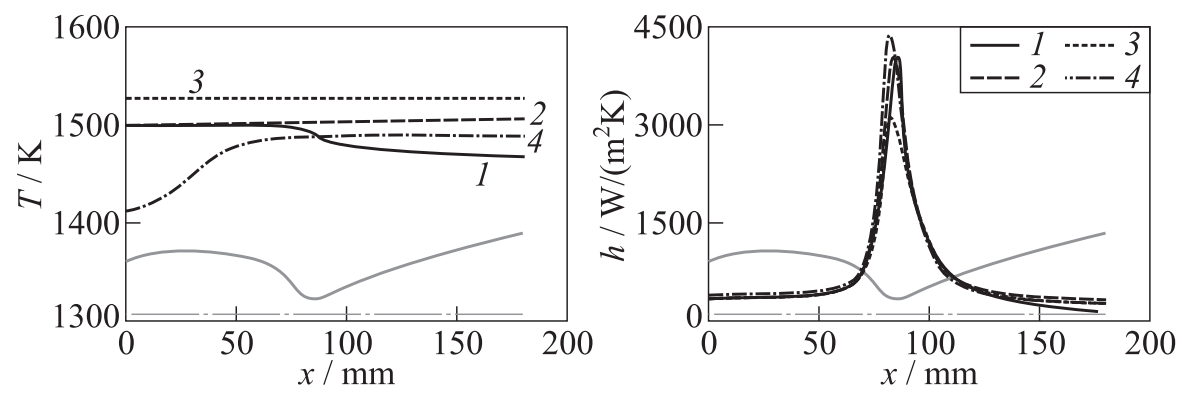

(a)
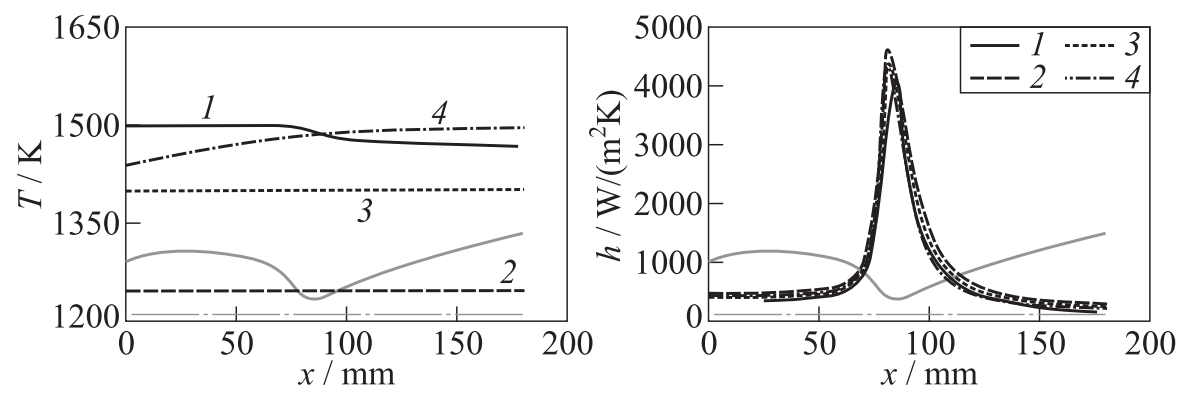

(b)

Figure 5 Boltzmann and Lorentz fitting for the Bartz heat load; uniform $(a)$ and variable $(b)$ wall thickness thrust chamber: reference $(1)$ and calculated $(2-1 \mathrm{~s} ; 3-$ $5 \mathrm{~s}$; and 4 - steady state) near-wall temperature and heat transfer coefficient

may be experimentally realized with a large combustion chamber and bleeding or tripping the boundary layer at the entrance of the expansion nozzle.

The fittings obtained for the near wall flow field temperature and the heat transfer coefficient for the Bartz heat load and a uniform thickness thrust chamber are shown in Fig. $5 a$. Figure $5 b$ shows the fittings for the variable wall thickness thrust chamber.

The agreement between the reference Bartz heat transfer coefficient profile and the Lorentz fitting is good. In the initial calculations, the merit function was evaluated using the temperature and heat transfer coefficient profiles along the whole length of thrust chamber. Under these conditions, the convergence of the method was poor and the value of the merit function at the end of the iterative process was very high. The main reason for this is that the Boltzmann and Lorentz families of fittings are not a "complete base" for the representation of the heat load.

Unlike the model problem, the imposed heat load is not guaranteed to have a profile that can be matched exactly by the fitting curves. Convergence to an exact solution is therefore not usually expected. 


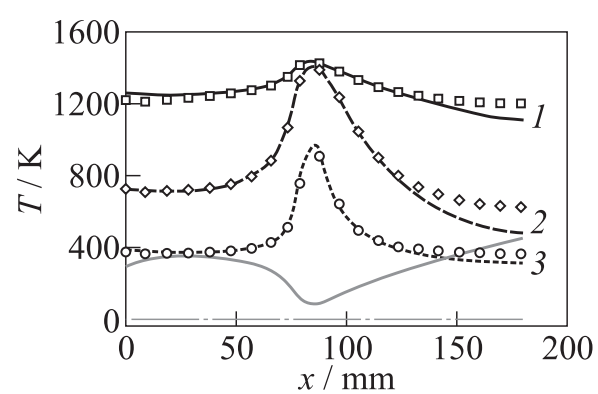

(a)

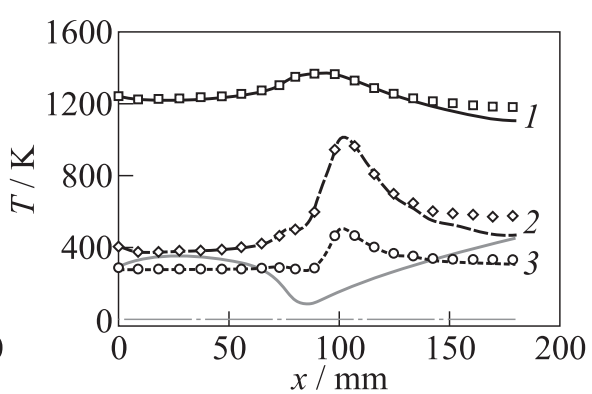

(b)

Figure 6 External wall temperature; Bartz reference curves and fitting curves; uniform $(a)$ and variable $(b)$ wall thicknesses: $1-$ steady state; $2-5 \mathrm{~s}$; and $3-1 \mathrm{~s}$

To improve results in the region of the high heat transfer rate, the domain of the evaluation of the merit function was clipped. The temperature data for the initial length of the chamber and the final part of the expansion nozzle were dropped from the calculation of the merit function.

The results for the near wall film temperature (the adiabatic wall temperature) were also in good agreement with the reference temperature. The results improved as the test time during the transient period increased. The best results are those for the steady state.

Even though the Boltzmann fitting would be able to represent the decreasing profile of the reference temperature, the recovered temperature profile was increasing along the length of the chamber.

The external temperature profile for the reference heat load and fitted data are shown in Fig. 6. First, the Bartz generated expressions for the adiabatic wall temperature and the heat transfer coefficient were used and the transient heat diffusion problem across the chamber wall was solved. The external wall temperature profile at two different time intervals during the transient period and in the steady-state regime are plotted as continuous lines in the Figure. These external wall temperature profiles were than used to apply the inverse method to obtain Boltzmann fitting for the adiabatic wall temperature and Lorentz fitting for the heat transfer coefficient. The heat diffusion problem across the wall was solved again, applying the heat load generated according to these fittings. The external temperature profiles for the same time intervals during the transient period and in the steady state regime were plotted using staggered symbols.

The effects of the data clipping in the evaluation of the merit function are evident in the external wall temperature profiles. Agreement between reference data and recovered data is better in the central part of the chamber; in the initial part of the chamber and near the end of the nozzle the values of the temperature for the two sets of data are considerably different. 


\subsection{Experimental Data}

In the third step, the "real experimental data" obtained from fire tests of a 400-newton bipropellant thruster were used to calculate the heat load.

The experimental data were obtained from a 400-newton bipropellant engine. The propellant pair is the NTO/MMH (Nitrogen Tetroxide/ Mono methyl Hydrazine). The test duration was $15 \mathrm{~s}$. The engine was tested with a mixture ratio in the range of $1.0: 1$ to $1.4: 1$. The data used for the analysis presented here were obtained from a test with mixture ratio of $1.1: 1$. The engine geometry and head construction are presented in [4]. The wall geometry is the variable wall thickness of Fig. 2.

The external wall temperature was recorded with a FLIR SYSTEMS infrared camera Model ThermaCAM SC3000. The maximum rate of recording is 30 frames per second with a grid of $320 \times 240$ pixels.

The pictures of the infrared images in three time intervals during the test are shown in Fig. 7

The distance between the viewing port and the thrust chamber was approximately $1.5 \mathrm{~m}$.
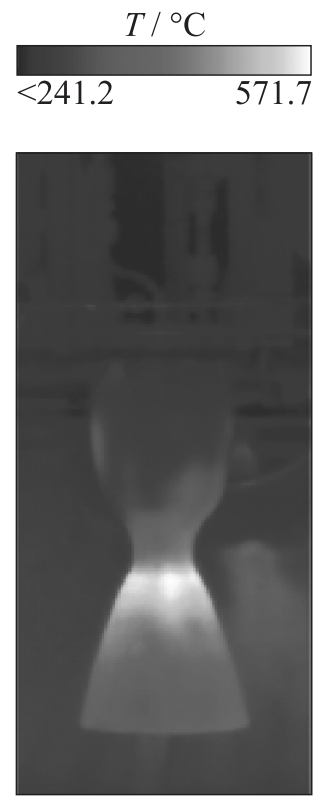

(a)
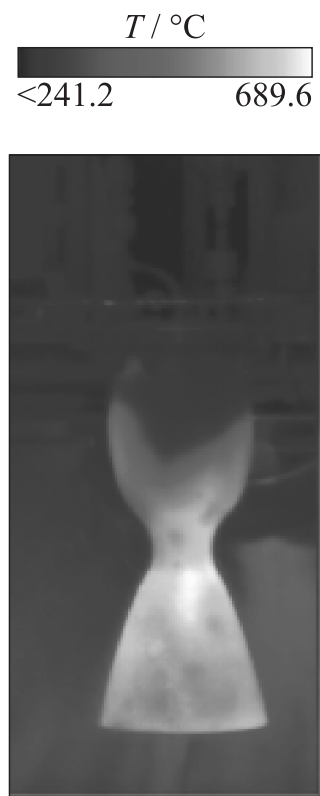

(b)
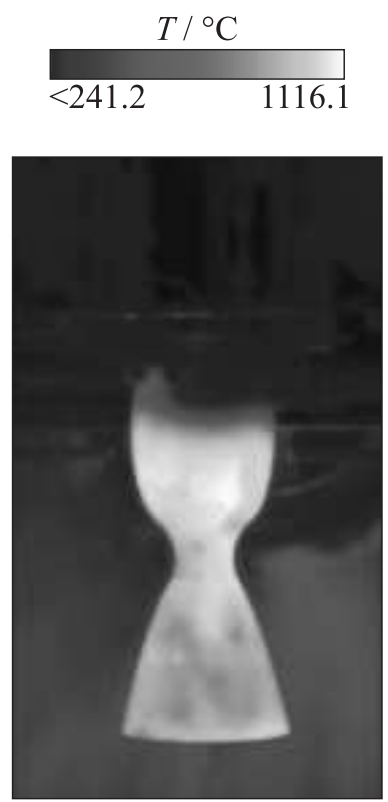

(c)

Figure 7 Infrared images of the thruster chamber wall temperature: $(a)$ at $5 \mathrm{~s} ;(b)$ at $10 \mathrm{~s}$; and $(c)$ at $20 \mathrm{~s}$ 
The camera was mounted outside the test chamber. The data were collected across a glass viewing port. The calibration of the camera was carried out in the following way.

First, the thrust chamber was mounted at a distance of $1.5 \mathrm{~m}$ in a direct view sight of the camera. The thrust chamber was heated by a gas blowing heat gun to the temperature up to $150{ }^{\circ} \mathrm{C}$. The readings of two thermocouples attached to the thrust chamber near the view sight were used as a reference value for the temperature. The emissivity parameter of the data processing unit of the camera was adjusted so that the temperature obtained by the camera was equal to the reference temperature from the thermocouple readings.

Next, the thrust chamber was mounted inside the vacuum chamber and the temperature data were taken with the infrared camera looking across the glass viewing port. The transmissivity (of the glass viewing port) parameter of the data processing software was adjusted.

Extending the range of the temperature in the calibration procedure and accounting for the window transmissivity variation for different wavelengths are high priority concern for the application of the method. In the calibrated range the estimated error in the temperature reading is appoximately $3{ }^{\circ} \mathrm{C}$.

The emissivity of the niobium aluminide protective layer being quite high $(\approx 0.6)$, the relative change with the temperature is generally low. For low emissivity materials, a change of emissivity by a factor of 2 or more would be possible. In this case, a calibration over the whole measurement range would be required.

The measured data were taken in 41 points equally distributed along the length of the chamber. At each position, the value of the temperature was averaged over an arc of approximately $30^{\circ}$, centered on the axis of the chamber.

The fittings obtained for the near wall flow field temperature and the heat transfer coefficient for the experimental heat load are shown in Fig. 8. The

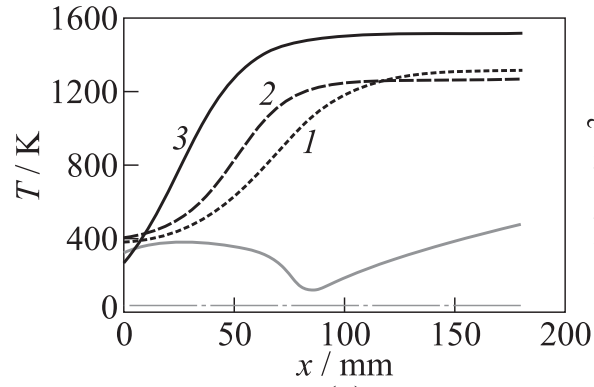

(a)

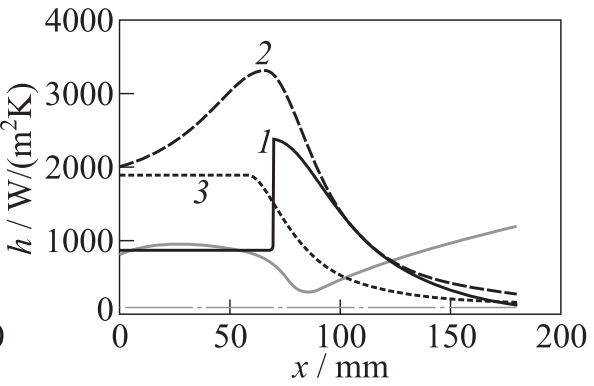

(b)

Figure 8 Boltzmann $(a)$ and Lorentz $(b)$ fittings for the experimental heat load; niobium variable wall thickness thrust chamber: $1-5 \mathrm{~s} ; 2-10$; and $3-15 \mathrm{~s}$ 
fittings were obtained from the wall temperature data in three time intervals: 5 , 10 , and $15 \mathrm{~s}$.

As with the Bartz model problem, the temperature data were clipped to a region near the throat of the chamber.

The Boltzmann temperature profile has the increasing value along the length of the thrust chamber as expected. The lower value of the temperature is approximately the same, but the upper value obtained from the 15 -second data is approximately $250{ }^{\circ} \mathrm{C}$ higher than the value obtained from the data of 5 and $10 \mathrm{~s}$. It is apparent that with increasing the time of the test the point of the maximum increase of the near wall gas temperature moves closer to the injector face of the engine.

The discrepancy in the Lorentz heat transfer coefficient profile was more pronounced. The maximum value was located near the throat, but the magnitude of the value varied by up to $50 \%$. Only the data from the 10 -second interval yielded a curve with a smooth maximum in the throat region. For the 5-and 15-second intervals, the heat transfer coefficient upstream of the throat was constant.

The main characteristics of the heat transfer coefficient Lorentz profiles are:

- $5 \mathrm{~s}$ : the FWHM of the left curve is negligibly small. Although not apparent in the figure, the line is still continuous with a continuous first derivative;

- 10 s: slightly different FWHM for the left and right curves. Visually smooth transition; and

- $15 \mathrm{~s}$ : the FWHM of the left side curve is much bigger than the length of the chamber, hence the apparent constant value of this part of the curve.

The behavior of both the Boltzmann fitting and the Lorentz fitting points to a conclusion that the heat load in the thrust chamber upstream of the throat region increases with time.

The most important information obtained from Fig. 8 is the temperature of the near-wall gas layer. This is the upper limiting value of the temperature of the chamber wall in steady state.

Figure $9 a$ shows the experimental surface temperature data and the temperature profiles obtained by the equivalent heat loads. The continuous lines show the measured temperature profiles along the lenghts of the thrust chamber at three different time intervals during the transient heating of the chamber. For each of these time intervals, the inverse problem was solved and a set of fittings was obtained for the Boltzmann and Lorentz curves. The transient heat diffusion problem in the chamber was solved again with the heat loads from the recovered data. The external temperature profile for the solutions is plotted again in the same figure using staggered symbols. It is quite remarkable that at each time interval the two temperature profiles are very close. 


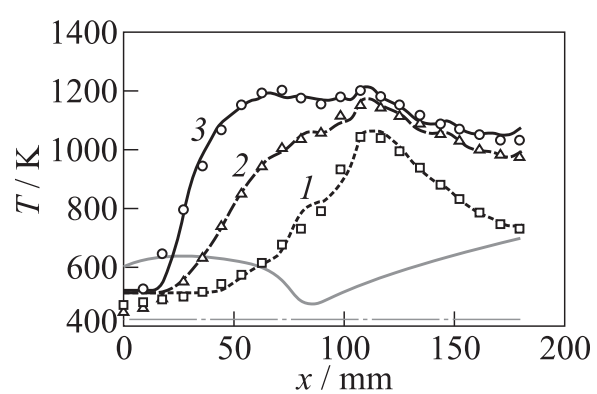

(a)

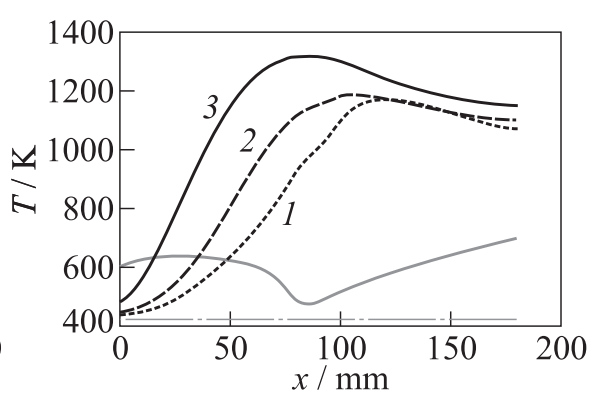

(b)

Figure 9 Surface temperature data, fittings data $(a)$ and extrapolation to steady state $(b): 1-5 \mathrm{~s} ; 2-10 ;$ and $3-15 \mathrm{~s}$

Figure $9 b$ shows the steady-state external wall temperature profile projected from the heat loads determined in three time intervals. The projected steadystate temperature of the supersonic part of the nozzle is very close to the heat load obtained in the three time intervals. In the combustion chamber and the subsonic part of the nozzle, the projected steady state temperature increases with the increase of the time interval of the data used to obtain the heat load.

This behavior contradicts the assumption that the the heat load does not change with the time. One possible reason is that the heating of the wall accelerates the evaporation of the fuel film near the wall and the reaction front moves upstream. It is also important to note that the heat load on the wall due to the radiation of the combustion products is not included in the calculations and this effect is more pronounced in the region upstream from the throat.

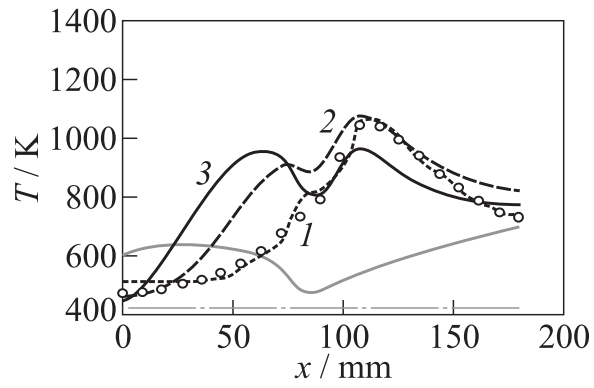

(a)

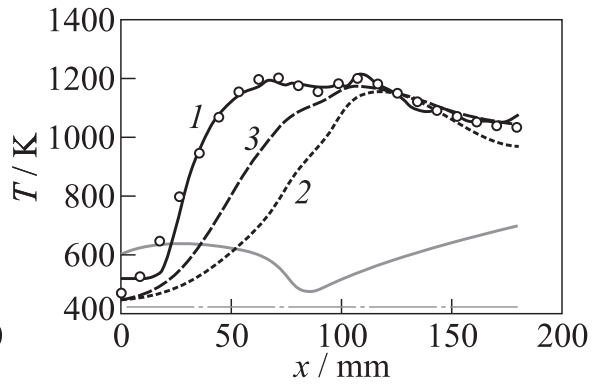

(b)

Figure 10 Surface temperature and cross extrapolation: $(a)$ measured $5 \mathrm{~s}(1)$ and extrapolated from $10(2)$ and $15 \mathrm{~s}(3)$; and $(b)$ measured $15 \mathrm{~s}(1)$ and extrapolated from $5 \mathrm{~s}(2)$ and $10 \mathrm{~s}(3)$ 
With respect to the experimental data used in the analysis, it must be observed that the axial symmetry of the heat load is poor. In the same axial position, there are azimuthal variation of the surface temperature and, therefore, of the heat load. This variation is not included in the solution of the heat conduction problem.

Figure 10 shows the measured temperature profile in two time intervals and the cross projected temperature profile from the heat load measured in the other time intervals. The time increasing heat load on the wall upstream from the throat is also evident in this figure. For the reference time interval, the supporting experimental data (continuous curve) and the temperature profile obtained from the implied heat load (staggered symbols) are plotted. For the projected temperature profiles at the other time intervals, only the temperature profile from the implied heat load are shown as continuous lines.

\section{CONCLUDING REMARKS}

A method to "see through" the thruster wall chamber using an inverse problem formulation is described. The method yields the heat load on the internal surface of the wall from the temperature measurement on the outside surface of the chamber.

A Boltzmann fitting is used to describe the temperature profile of the near wall gas flow temperature (the adiabatic wall temperature). A Lorentz fitting is used to describe the heat transfer coefficient profile along the length of the thrust chamber.

A model problems is used to verify the self-consistency of the algorithm employed to obtain the fittings.

The analytical solution due to Bartz is used to test the capability of the curve fittings to capture the expected profile of the heat load along the length of the thrust chamber.

The experimental validation of the method is done by using test data from the fire test of a 400-newton bipropellant engine. The test engines use a swirl injector head plate and a radiation cooled thrust chamber.

The analysis of the data obtained suggests a time increasing heat load upstream of the chamber nozzle throat.

For further improvement of the method, curve fittings with better "base completeness" and "orthogonality characteristics" are desirable.

The accuracy of the experimental data, especially in the high range temperature, also needs the confidence in the results of the analysis. 


\section{ACKNOWLEDGMENTS}

This project was supported by FAPESP PN 2003/06878-6 and CNPq PN 303359/2006-4.

\section{REFERENCES}

1. Press, W. H., S. A. Teukolsky, W. T. Vetterling, and B. P. Flannery. 1999. Numerical recipes in C. 2nd ed. Cambridge University Press.

2. Hill, P. G., and C. R. Peterson. 1970. Mechanics and thermodynamics of propulsion. Reading, MA, USA: Addison Wesley Publ. Co.

3. Huzel, D. K., and D.H. Huang. 1992. Modern engineering for design of liquidpropellant rocket engines. Washington, DC, USA: AIAA.

4. Hinckel, J. 2011. A $400 \mathrm{~N}$ bipropellant thruster with swirl injectors. 4th European Conference for Aerospace Sciences. St.-Petersburg. No. 2011-563. 